\title{
Labyrinthe
}

13 | 2002

Numéro 13

Traduction

\section{Le débat sur 1948}

Avi Shlaim

\section{(2) OpenEdition}

Journals

Édition électronique

URL : http://journals.openedition.org/labyrinthe/1509

DOI : $10.4000 /$ labyrinthe.1509

ISSN : 1950-6031

Éditeur

Hermann

Édition imprimée

Date de publication : 15 novembre 2002

Pagination : 81-110

Référence électronique

Avi Shlaim, « Le débat sur 1948 », Labyrinthe [En ligne], 13 | 2002, mis en ligne le 04 mai 2007, consulté le 19 avril 2019. URL : http://journals.openedition.org/labyrinthe/1509; DOI : 10.4000/labyrinthe.1509

Ce document a été généré automatiquement le 19 avril 2019

Propriété intellectuelle 


\title{
Traduction
}

\section{Le débat sur 1948}

\author{
Avi Shlaim
}

Source : « The Debate about 1948 », dans International Journal of Middle East Studies, vol. 27, $\mathrm{n}^{\circ}$ 3, août 1995, pp. 287-304. Reproduit dans Ilan Pappé (dir.), The Israel/Palestine Question, Londres et New York, Routledge, 1999 Traduction de Philippe Bourmaud

1 «Les conquérants, mon garçon, ne considèrent comme vraie que l'histoire qu'ils ont euxmêmes fabriquée ${ }^{1}$. » C'était la remarque que faisait le vieux maître d'école au jeune Saïd à son retour à Haïfa pendant l'été 1948, dans le roman tragi-comique d'Émile Habibi, Les Aventures de Sa'îd le Peptimiste. L'enseignant parlait des Israéliens avec plus de chagrin que de colère : « Il est vrai qu'ils ont démoli ces villages... et chassé leurs habitants. Mais, mon garçon, ils sont bien autrement cléments que les conquérants que furent nos aïeux il y a tant d'années ${ }^{2}$. »

2 La plupart des Israéliens seraient furieux qu'on les considère comme des conquérants mais c'est bien ainsi qu'ils sont perçus par les Palestiniens. Toutefois, l'intérêt de cette citation est plutôt de voir qu'il ne peut y avoir d'accord sur ce qui s'est vraiment passé en 1948 ; chaque camp souscrit à une version différente des événements. Les Palestiniens considèrent les Israéliens comme des envahisseurs et se perçoivent comme les vraies victimes de la première guerre israélo-arabe, qu'ils appellent al-Nakba, autrement dit la catastrophe. L'historiographie palestinienne reflète ces perceptions. Les Israéliens, qu'ils aient ou non été des conquérants, furent les vainqueurs incontestés de la guerre de 1948, qu'ils appellent la guerre d'Indépendance. Du fait de leur victoire, entre autres raisons, ils furent en mesure de diffuser plus efficacement que leurs adversaires leur version de cette guerre tragique. L'histoire, en un sens, est la propagande des vainqueurs.

Le récit sioniste traditionnel de la guerre de 1948 est grosso modo le suivant. Le conflit entre Juifs et Arabes en Palestine entra dans une phase critique à la suite de l'adoption, le 29 novembre 1947, de la résolution des Nations unies imposant la partition du territoire et demandant l'instauration de deux États, l'un juif et l'autre arabe. Les Juifs acceptèrent 
le plan de l'ONU en dépit des sacrifices pénibles qu'il impliquait, mais les Palestiniens, les États arabes voisins et la Ligue arabe le rejetèrent. La Grande-Bretagne fit tout ce qui était en son pouvoir, dans les derniers moments du Mandat sur la Palestine, pour faire obstacle à la création de l'État juif envisagé par le plan. Lorsque expira le Mandat et que l'État d'Israël fut proclamé, sept États arabes envoyèrent leurs armées en Palestine avec la ferme intention d'étouffer l'État juif dès sa naissance. Le conflit qui s'ensuivit était inégal, et opposait un David juif et un Goliath arabe. L'État juif au berceau livra une bataille désespérée, héroïque, et, finalement, victorieuse, pour sa survie, et ce contre toute probabilité. Pendant la guerre, des centaines de milliers de Palestiniens fuirent vers les États arabes voisins, principalement en réponse aux ordres de leurs chefs et malgré les appels des Juifs pour les faire rester et démontrer qu'une coexistence pacifique était possible. Après la guerre, d'après ce même récit, les dirigeants israéliens tentèrent de tout leur cœur et de toute leur force de conclure la paix mais ils ne trouvèrent personne avec qui discuter. L'intransigeance des Arabes fut seule responsable de l'impasse politique dont il fut impossible de sortir avant la visite du président Anwar al-Sadat à Jérusalem, trente ans plus tard.

4 Ce récit sioniste traditionnel, ou histoire de la «vieille école» de la guerre de 1948, présente plusieurs facettes. En premier lieu, ce n'est pas de l'histoire au sens propre. L'essentiel de l'abondante littérature sur la guerre fut écrit, non par des historiens professionnels, mais par des personnes impliquées dans le conflit, hommes politiques, soldats, historiens officiels, et un large contingent de chroniqueurs, journalistes, biographes et hagiographes, tous partisans. En deuxième lieu, cette littérature est très limitée sur l'analyse politique de la guerre et s'étend longuement sur la chronique des opérations militaires, tout spécialement les actions héroïques des combattants israéliens. En troisième lieu, elle affirme que la conduite d'Israël fut, tout au long de la guerre, guidée par des exigences morales plus élevées que celles de ses ennemis. Il est particulièrement à propos d'évoquer le précepte de tohar haneshek, à savoir la pureté des armes, qui stipule que les armes restent pures pourvu qu'elles ne servent qu'à des fins d'autodéfense, et qu'elles ne soient pas employées contre des civils innocents ni des populations désarmées. Cette version de l'épopée morale de la guerre de 1948 est celle qui est enseignée dans les écoles israéliennes et qui est largement utilisée à l'étranger dans un but de légitimation. C'est un exemple caractéristique de l'utilisation d'une version nationaliste de l'histoire dans un processus de construction nationale.

Jusque récemment, cette version sioniste admise des événements constituant le contexte de la naissance de l'État d'Israël n'avait à peu près pas été contestée hors du monde arabe. Le quarantième anniversaire de la création de l'État, cependant, donna lieu à la publication d'une série d'ouvrages qui remettaient en cause différents aspects de ce récit traditionnel. Le premier et le plus complet dans sa variété de perspectives, fut, sur un ton polémique, le livre de Simha Flapan, The Birth of Israel: Myths and Realities. Flapan, qui avait été, au sein du parti de gauche radical Mapam, directeur du département des affaires arabes, et occupait la fonction de rédacteur en chef du mensuel sur le MoyenOrient New Outlook, écrivit explicitement son livre avec une intention politique plutôt qu'académique: révéler les mythes qui, affirmait-il, constituaient la base de la propagande israélienne et de la ligne politique de l'État hébreu. «Les mythes qu'Israël a forgés pendant la formation de l'État, écrit-il, se sont consolidés jusqu'à devenir un impénétrable et dangereux bouclier idéologique ${ }^{3}$. " Après avoir relevé sept mythes, qu'il traite chacun dans un chapitre particulier, Flapan admet en toute franchise l'objectif 
politique de tout ce travail. «Le but de ce livre est de discréditer ces mythes, non pour des motivations académiques, mais pour contribuer à une meilleure compréhension du problème palestinien et à une approche plus constructive des tentatives faites pour le résoudre ${ }^{4}$.»

Parmi les autres livres qui, par leur traitement du compte rendu sioniste des événements, s'inscrivent dans une perspective critique mais sans intention politique explicite, il faut compter celui de Benny Morris, The Birth of the Palestinian Refugee Problem, 1947-19495 ; celui d'Ilan Pappé, La Guerre de 1948 en Palestine. Aux origines du conflit israélo-arabe ${ }^{6}$; et le mien, Collusion across the Jordan: King Abdullah, the Zionist Movement and the Partition of Palestine ${ }^{7}$. Ensemble, nous fûmes désignés comme les révisionnistes israéliens ou les "nouveaux historiens ». Aucun de ces deux termes n'est entièrement satisfaisant. Le terme "révisionnistes ", dans le lexique sioniste, renvoie déjà aux radicaux de droite disciples de Ze'ev Jabotinsky qui quittèrent le courant dominant du sionisme en 1925, alors que les nouveaux historiens se situent sur l'échiquier politique quelque part sur la gauche du courant dominant. Le terme "nouveaux historiens" est plutôt auto-complaisant, et rejette implicitement comme vieux et sans valeur tout ce qui a été écrit avant qu'euxmêmes n'apparaissent sur la scène. Le Pr Yehoshua Porath de l'université hébraïque de Jérusalem a suggéré, en guise d'alternative, les mots "préhistoire » et " histoire ». Ce n'est toutefois qu'une atténuation mineure apportée à l'insulte faite à la première catégorie d'historiens. Par conséquent, et faute d'une meilleure expression, j'utiliserai l'étiquette "vieille école » en référence aux partisans de la version sioniste admise de la guerre de 1948 et le label «nouveau » pour les travaux récents des critiques de gauche de cette version, moi y compris.

7 La première remarque à faire sur la nouvelle historiographie est que, pour une bonne part, elle n'est pas nouvelle. Nombre des arguments qui sont au cœur de la nouvelle historiographie ont été soutenus depuis longtemps par des auteurs israéliens, sans parler de leurs confrères palestiniens, arabes et occidentaux. Dresser la liste des auteurs israéliens dépasse le cadre de cet article, mais il convient de donner quelques exemples. Un des fils directeurs de la nouvelle historiographie est une position critique à l'égard de David Ben Gourion, le fondateur de l'État d'Israël et son premier chef de gouvernement. Alors que les historiens de la vieille école ont tendance à voir en Ben Gourion le représentant du consensus au sein des élites civiles et militaires, les nouveaux historiens sont enclins à le décrire comme l'aiguillon qui a guidé la politique israélienne en 1948, et en particulier l'expulsion des Palestiniens. Au reste, nombre des critiques récentes à l'égard de Ben Gourion sont déjà entr'aperçues dans un livre écrit par l'ancien historiographe officiel des Forces de défense israéliennes ${ }^{8}$, le lieutenant-colonel Israël Baer, durant sa détention pour espionnage au profit de l'Union soviétique9.

8 La révision de la version traditionnelle de la politique britannique à la fin du Mandat fut entamée de façon significative par Gavriel Cohen dans l'article publié dans le livre au titre indéniablement suranné - Hayinu Keholmim ${ }^{10}$ (Nous étions des rêveurs). Yaacov Shimoni, directeur adjoint du département Moyen-Orient du ministère des Affaires étrangères en 1948 publia un article très pénétrant sur les hésitations, doutes, réserves et opinions contradictoires qui accompagnaient la décision des Arabes d'intervenir en Palestine en mai $1948^{11}$. Cet article, qui contredit le récit sioniste dominant, est d'autant plus remarquable qu'il a été écrit par un homme du sérail. Meir Pa'il apporta un autre correctif à la vision d'un monde arabe monolithique, en se concentrant tout particulièrement sur le conflit entre le roi Abdallah de Jordanie et les Palestiniens ${ }^{12}$. La 
version sioniste des causes du problème des réfugiés palestiniens a été mise en doute par bon nombre d'auteurs israéliens, et, de façon tout spécialement convaincante, par Rony $\mathrm{E}$. Gabbay ${ }^{13}$. Enfin, l'argument selon lequel les efforts réels d'Israël pour parvenir à une paix avec les Arabes n'étaient pas à la mesure de la rhétorique officielle, peut être rattaché à un livre publié sous un pseudonyme par deux membres du parti communiste israélien ${ }^{14}$.

Bien que de nombreux arguments de la nouvelle historiographie ne soient pas en euxmêmes tout neufs, il y a une différence qualitative entre celle-ci et l'ensemble des études antérieures, qu'elles aient accepté ou contredit la ligne officielle sioniste. La différence, en bref, est que la nouvelle historiographie a été écrite avec la possibilité d'accéder aux documents officiels israéliens et occidentaux, alors que les auteurs antérieurs n'en ont pas eu le droit, ou n'ont eu qu'un droit d'accès partiel. Ce n'est pas une règle sans exception; il est autant de dérogations que de degrés d'accessibilité. Néanmoins, il est généralement exact de dire que les nouveaux historiens, à l'exception de feu Simha Flapan, ont mené un vaste travail d'archives en Israël, au Royaume-Uni et aux États-Unis, et que leurs points de vue sont appuyés sur des preuves documentaires solides et un appareil critique à l'occidentale.

De fait, le surgissement de ces nouvelles histoires n'eût pas été possible tant que les documents gouvernementaux officiels n'avaient pas été déclassifiés. Israël a repris la règle des trente ans en vigueur en Grande-Bretagne en ce qui concerne les archives de politique étrangère. Si cette règle ne s'étend pas à tous les documents de façon aussi systématique qu'au Royaume-Uni, on constate en revanche davantage de libéralité dans son application. La Grande-Bretagne et Israël, l'un comme l'autre, ont commencé à suivre le modèle américain, et à publier des recueils de documents sélectionnés et annotés par des professionnels. Les quatre premiers volumes des séries Documents on the Foreign Policy of Israel sont d'une utilité inestimable et constituent l'appui indispensable à toute recherche sur la guerre de 1948 et sur les négociations d'armistice qui y ont mis fin ${ }^{15}$.

11 Du côté arabe, il n'y a pas d'équivalent à cette règle des trente ans. Dans les fonds d'archives concernés, l'accès aux documents sur la guerre de 1948 est très limité, et cela pose un problème sérieux au chercheur. On affirme souvent qu'aucune version définitive de la guerre de 1948, et surtout de ce qui s'est passé en coulisse du côté arabe, n'est possible sans un accès effectif aux archives d'État arabes. Cela étant, cette difficulté ne signifie pas qu'il y ait impossibilité. Tout d'abord, certains documents arabes officiels sont disponibles. Un bon exemple est le rapport du comité parlementaire irakien chargé de l'enquête sur la question de Palestine, qui est rempli de documents émis à un haut niveau de responsabilités ${ }^{16}$. Un autre exemple est la collection de papiers officiels, semi-officiels et privés réunis par l'Institut d'études palestiniennes ${ }^{17}$. En outre, il existe en arabe une littérature non négligeable, constituée de récits de première main de la catastrophe, et cela inclut les journaux et mémoires d'hommes politiques et de militaires de premier plan 18. Et quand bien même aucune de ces sources arabes n'existerait, les autres sources disponibles fourniraient encore la base d'une analyse bien informée de la guerre de 1948. Un historien militaire du Moyen Âge pâlirait d'envie à la vue de la masse des sources à la disposition de son confrère spécialiste du Moyen-Orient. Les historiens de la guerre de 1948 feraient bien mieux d'explorer en profondeur les multiples sources qui leur sont accessibles, plutôt que de se plaindre du refus d'accès qui leur est opposé par les fonds d'archives d'État arabes.

12 Si la mise à disposition de sources d'information, nouvelles et riches, a été l'une des raisons de l'apparition de ce révisionnisme historique, un certain changement du climat 
politique général en fut une autre ${ }^{19}$. Pour de nombreux Israéliens, en particulier les plus libéraux d'entre eux, l'invasion du Liban en 1982, décidée par le Likoud, mal conçue et promise à bien des tragédies, a marqué un tournant. Jusqu'alors, les dirigeants sionistes avaient pris soin de cultiver l'image d'un peuple pacifique qui ne part à la guerre que lorsqu'elle leur est imposée. Jusqu'alors, la notion d'ein breira (pas d'alternative), était cruciale pour toute explication des raisons pour lesquelles Israël prenait les armes, et constituait un moyen de légitimer ses interventions militaires. Pourtant, alors même qu'un débat féroce faisait rage entre partisans et adversaires de la guerre du Liban, le Premier ministre

13 Menachem Begin donna une conférence à l'Académie supérieure de Tsahal sur les guerres délibérées et les guerres imposées. Il affirma que la guerre du Liban, comme la guerre du Sinaï en 1956, était une guerre délibérée, conçue pour mener à bien des objectifs nationaux. À partir de cet aveu, sans précédent dans l'histoire du mouvement sioniste, le consensus national bâti sur la notion d'ein breira commença à s'effondrer, libérant un espace politique pour un réexamen critique de l'histoire antérieure du pays ${ }^{20}$.

La parution des nouveaux ouvrages sur la guerre de 1948 suscita beaucoup d'intérêt et de controverses dans les cercles universitaires et politiques israéliens. En avril 1989, une conférence de deux jours, consacrée à la guerre d'Indépendance et organisée par le Centre Dayan et l'Institut de recherches sionistes à l'université de Tel-Aviv, tourna à la confrontation entre la version sioniste de la vieille école représentée par des historiens, des journalistes et des vétérans de cette guerre, et la nouvelle version représentée par Benny Morris et moi-même. Plusieurs des intervenants arguaient, avec quelque raison, que les nouveaux historiens ne produisaient pas une nouvelle école, ni une nouvelle méthodologie de l'écriture historique, mais utilisaient des méthodes historiques conventionnelles pour soutenir de nouvelles interprétations de la guerre de 1948. Sur les mérites de ces nouvelles interprétations, les opinions étaient violemment divisées. Des membres de la vieille garde, et particulièrement de celle du Mapaï, faisaient des sorties hostiles et condamnaient indistinctement le tout. La réponse de la communauté universitaire israélienne, tant à la conférence que dans les recensions et discussions ultérieures, fut plus mesurée. Certaines des découvertes de la nouvelle historiographie, et en particulier celles exposées dans le livre de Benny Morris, furent, au fil du temps, largement acceptées dans la communauté scientifique israélienne et apparurent même dans les bibliographies des étudiants et les manuels de lycée.

Parmi les critiques des nouveaux historiens, le plus violent et le plus porté sur le vitriol fut Shabtai Teveth, le biographe de Ben Gourion. Son attaque intitulée «Les nouveaux historiens ", parut en pleine page dans quatre numéros du quotidien israélien Ha'aretz, des 7, 14 et 21 avril, et du 19 mai 1989. Cet auteur publia par la suite une version revue et abrégée de cette série sous la forme d'un article intitulé «Charging Israel with Original Sin» dans le mensuel juif américain Commentary. Dans cet article, Teveth décrit la nouvelle histoire comme "un ramassis de distortions, d'omissions, de lectures tendancieuses, et de falsifications pures et simples ${ }^{21} »$. Teveth livrait bataille sur deux fronts. D'une part, il attaquait la nouvelle historiographie en affirmant qu'elle « repose en partie sur des éléments de preuve défectueux, et se caractérise par de sérieuses déficiences professionnelles ${ }^{22}$ ». D'autre part, il avançait que cette historiographie était orientée politiquement et pro-palestinienne, et visait à délégitimer le sionisme et l'État d'Israël. 
16 À l'appui de ces assertions, Teveth cite un passage de l'article de Benny Morris sur « La nouvelle historiographie », passage qui énonce que «la manière dont on perçoit 1948 pèse lourdement sur la perception que l'on a de toute l'expérience sioniste/israélienne... Si Israël est né dans la faute, sali par un péché originel, alors ce pays ne méritait pas plus les grâces et l'assistance [de l'Occident] que ses voisins. » Teveth affirme ensuite que le péché originel dont Shlaim inculpe Israël consiste « à refuser aux Arabes palestiniens de disposer d'un pays », tandis que Morris impute à Israël la «création du problème des réfugiés ", et l'une et l'autre accusations " sont fausses " $^{23}$.

17 Teveth a dû parcourir les deux ouvrages en question avec un peigne extrêmement fin pour découvrir la preuve des motivations politiques qu'il prête à ces auteurs, mais même ainsi il est rentré bredouille. C'est pourquoi il a été réduit à citer l'article tiré de Tikkun, qu'il reconstruit, en un ramassis de distorsions de son propre cru, pour en faire le manifeste politique de ce qu'il appelle «le nouveau club historique ». Cependant, même les citations de l'article ne manifestent aucun but politique ; l'auteur ne fait que mettre en lumière les attitudes occidentales à l'égard d'Israël, qui sont influencées par la perception des circonstances de la création d'Israël. C'est à n'en pas douter un point incontestable. Benny Morris répondit dans Ha'aretz et dans un second article publié dans Tikkun que, en ce qui le concernait, la nouvelle historiographie n'avait nul but politique de quelque sorte que ce soit. La tâche et la fonction de l'historien, de son point de vue, sont d'éclairer le passé24. Ma propre opinion est que la tâche la plus fondamentale de l'historien n'est pas de tenir une chronique, mais d'évaluer. La tâche de l'historien est de soumettre les affirmations de tous les protagonistes à une analyse rigoureuse et de rejeter toutes celles, même les plus révérées, qui ne résistent pas à cet examen. À mon avis, nombre des affirmations des historiens de la vieille école ne résistent pas à un examen sérieux. Cela ne veut pas dire que tout ce qu'ils affirment est erroné ou qu'Israël est le seul méchant de la pièce. En fait, ni Benny Morris ni moi, nous n'avons chargé Israël d'un péché originel. C'est Shabtai Teveth qui, en dépit de toutes les preuves du contraire, continue à s'accrocher à la doctrine de l'immaculée conception d'Israël ${ }^{25}$.

18 C'est la contre-attaque de Teveth qui est orientée politiquement. Comme beaucoup d'autres membres de la vieille garde du Mapaï, il ne sait distinguer entre l'histoire et la propagande. Toute tentative de réviser la sagesse traditionnelle sous les lumières de nouvelles preuves exhumées par l'historien est donc immédiatement suspecte, antipatriotique, calculée pour faire du tort à la réputation du dirigeant et du parti qui menèrent la lutte pour l'indépendance. Pour Teveth et certains membres de la vieille garde du Mapaï, les événements en question n'appartiennent pas complètement à l'histoire mais représente l'heure de gloire de leur parti et de leur pays. Il sont trop attachés, personnellement et politiquement, à la version héroïque de la création de l'État d'Israël pour pouvoir traiter la nouvelle historiographie avec un esprit ouvert.

De façon intéressante, des personnalités à droite de l'échiquier politique israélien, universitaires et autres, ont répondu à ces découvertes avec bien davantage d'équanimité. Elles admettent sans difficulté, par exemple, qu'Israël a bel et bien expulsé les Palestiniens et expriment même des regrets que cet État n'en ait pas expulsé un nombre plus grand encore, puisque c'étaient eux qui avaient déclenché la guerre. Les radicaux de droite ont tendance à considérer la guerre de 1948 d'un point de vue empreint de realpolitik, plutôt que du point de vue de la moralité. Ils s'épargnent ainsi l'angoissante tâche de tenter de réconcilier les pratiques du sionisme avec les principes du libéralisme. C'est peut-être pour cette raison qu'ils sont généralement moins sûrs de leur fait et plus 
réceptifs à de nouvelles informations et à des analyses inédites de la guerre de 1948 que les membres de la vieille garde du Mapaï. Ces derniers accordent tant d'importance à la prétention d'Israël à la rectitude morale, qu'ils ne peuvent faire face aux preuves manifestes du double jeu cynique des gouvernants d'Israël ou à l'expulsion brutale et à la dépossession des Palestiniens. C'est un des postulats de base de leur récit qu'Israël est la victime innocente. C'est également leur souci des conséquences politiques d'une réécriture de l'histoire qui rend compte, pour l'essentiel, de la férocité de leurs attaques contre la nouvelle historiographie.

Bien que la politique et l'histoire se soient trouvées emmêlées dans le débat sur 1948, et bien que ce débat ressemble souvent à un dialogue de sourds, le fait même qu'un débat ait lieu est un agréable changement par rapport à l'étouffante conformité du passé. A.J.P. Taylor faisait remarquer que l'histoire ne se répétait pas, mais que les historiens, eux, se répétaient les uns les autres. La « vieille » historiographie de l'émergence d'Israël est un exemple frappant de ce phénomène général. Pour ce qui est de la nouvelle historiographie, et quels que soient ses défauts, elle a au moins le mérite de pousser à un réexamen des conventions narratives sanctifiées par les années.

Six pommes de discordes peuvent être identifiées dans les débats actuels entre «nouveaux » historiens et historiens de la vieille école : la politique britannique à la fin du Mandat, le rapport de forces entre Israël et les Arabes en 1948, les origines du problème des réfugiés palestiniens, la nature des relations israélo-jordaniennes pendant la guerre, les buts de guerre arabes, et les motifs de l'impasse politique durable après l'arrêt des combats. Qu'il me soit permis de rendre compte brièvement des principaux arguments et contre-arguments sur ces six thèmes clés du débat, et que l'on garde à l'esprit que je ne suis pas un observateur détaché ou neutre, mais l'un des protagonistes du débat.

La politique britannique

Le premier motif d'opposition concerne la politique britannique en Palestine entre le 29 novembre 1947 et le 14 mai 1948. L'historiographie sioniste, qui reflète les soupçons des dirigeants sionistes à l'époque, est pleine d'accusations de complots hostiles censés avoir pesé contre le Yishouv ${ }^{26}$ pendant le crépuscule de la domination anglaise en Palestine. L'accusation principale veut que la Grande-Bretagne ait armé et secrètement encouragé ses alliés arabes, tout particulièrement son client, le roi Abdallah de Jordanie, les poussant à envahir la Palestine lorsque expirerait le mandat britannique, et à combattre l'État juif dès sa naissance. C'est à Ernest Bevin, à la tête du Foreign Office dans le gouvernement travailliste dirigé par Clement Attlee, qu'est dévolu le rôle de grand méchant dans cette conspiration supposée.

Ilan Pappé, au moyen de sources en anglais, en arabe et en hébreu, a taillé à la hache à travers la présentation sioniste traditionnelle de la politique britannique à la fin du Mandat, et j'ai essayé de le suivre sur cette voie nouvelle ${ }^{27}$. La clé pour comprendre la politique britannique pendant cette période est résumée en deux mots par Pappé : la grande Transjordanie. Bevin avait le sentiment que, s'il fallait partager la Palestine, la partie arabe ne devait pas être indépendante, mais serait rattachée à la Transjordanie. Une grande Transjordanie compenserait aux yeux de la Grande-Bretagne la perte de ses bases en Palestine. L'hostilité à Hajj Amin al-Husayni ${ }^{28}$, qui avait lié son sort à celui des nazis pendant la Seconde Guerre mondiale, l'hostilité à l'existence d'un État palestinien, qui dans l'esprit des Britanniques serait toujours l'État du mufti, ont été des caractéristiques essentielles et constantes de la politique britannique après-guerre. En 
février 1948, Bevin et ses conseillers du Foreign Office s'étaient réconciliés, par pragmatisme, avec l'idée de l'apparition inévitable de l'État juif. Ce qui ne passait pas, c'était l'émergence d'un État palestinien.

La politique de la grande Transjordanie impliquait un soutien discret à une demande d'Abdallah - surnommé «le petit roi de M. Bevin» par les fonctionnaires du Foreign Office -, afin d'élargir son royaume en s'emparant de la Cisjordanie. Lors d'une rencontre secrète à Londres le 7 février 1948, Bevin donna au Premier ministre transjordanien Tawfiq Abul Huda le feu vert pour l'envoi de la Légion arabe en Palestine, juste après le départ des troupes britanniques. Cependant, Bevin donna ordre à la Jordanie de ne pas envahir la zone octroyée par les Nations unies aux Juifs. Une attaque contre le territoire de l'État juif, disait-il, forcerait la Grande-Bretagne à supprimer son assistance financière à la Légion arabe et à en retirer ses officiers. Loin d'être guidés par des préjugés antisémites aveugles, au point de lancer la Légion arabe contre les Juifs, Bevin insista en fait lourdement sur la modération des Arabes en général et de la Jordanie en particulier. Quelques péchés que le secrétaire britanniques aux Affaires étrangères ait commis sur la fin sans gloire du Mandat, on ne peut y ajouter celui d'avoir incité le roi Abdallah à se servir de la force pour empêcher la formation d'un État juif.

Si Bevin fut coupable d'avoir conspiré dans le but de lancer les opérations de la Légion arabe, son objectif n'était pas les Juifs mais les Palestiniens. La perspective d'un État palestinien était de toute façon assez lointaine, puisque les Palestiniens eux-mêmes avaient fait si peu pour l'édifier. Cependant, en soutenant les prétentions d'Abdallah à la prise de la partie arabe de la Palestine qui se trouvait adjacente à son royaume, Bevin contribuait à faire en sorte que l'État palestinien prévu par le plan de partition de l'ONU reste mort-né. Pour faire bref, s'il faut accuser Bevin, ce n'est pas de s'être efforcé de faire avorter l'État juif mais d'avoir approuvé l'accord entre le roi Abdallah et l'Agence juive, qui prévoyaient de se partager la Palestine et de laisser les Palestiniens au dehors et tout à leur malheur.

L'accusation sioniste, selon laquelle Bevin aurait délibérément organisé les hostilités en Palestine et fourni aux Arabes tous les encouragements et toutes les armes nécessaire pour écraser l'État juif à peine créé, est presque l'opposé exact de la vérité historique, telle qu'elle ressort des documents britanniques, arabes et israéliens. L'argument manque de substance et peut sans risque être relégué, le premier, parmi les mythes qui ont entouré la création de l'État d'Israël.

L'équilibre des forces militaires

27 Un deuxième mythe, nourri par des récits officiels et semi-officiels de la guerre de 1948, est que la victoire israélienne fut obtenue en dépit d'une insurmontable infériorité en terme de capacité militaire. Israël est décrit dans ces sources comme un petit David juif affrontant un gigantesque Goliath arabe. La guerre est décrite comme une lutte désespérée, coûteuse et héroïque pour la survie, dans laquelle Israël jouerait le rôle du petit costaud chassant les armées de brigandages envoyées par sept États arabes. La victoire finale d'Israël dans cette guerre n'est vue, ni plus ni moins, que comme un miracle.

Il ne s'agit pas de mettre en question l'héroïsme des combattants juifs, et il n'y a pas de doute que le tribu payé par le Yishouv pour sa victoire fut lourd. En fin de compte, il y eut 6000 morts, -4000 soldats et 2000 civils - , soit environ $1 \%$ de la population totale. Cependant, le Yishouv n'était pas dans une infériorité numérique et matérielle aussi désespérée que l'historiographie officielle veut bien nous le faire croire. Il est vrai que le 
Yishouv était peuplé de tout juste 650000 âmes, à comparer aux 1,2 million d'Arabes palestiniens et aux quelques quarante millions d'Arabes dans les États voisins. Il est vrai que les conseillers militaires de plus haut rang annoncèrent le 12 mai 1948 à la direction politique que la Haganah n'avait qu'une probabilité «à cinquante-cinquante» de repousser l'attaque arabe imminente. Il est vrai que le sentiment de faiblesse et de vulnérabilité de la population juive était aussi aigu que généralisé, et que certaines fractions de cette population furent prises d'un sentiment de ténèbres et d'apocalypse. Il est encore vrai que pendant trois semaines critiques, de l'invasion de la Palestine par les armées régulières arabes le 15 mai, jusqu'au début de la première trêve le 11 juin, cette communauté était en lutte pour sa survie.

Pourtant, le Yishouv jouissait également d'un certain nombre d'avantages que les historiens de la vieille école ont généralement sous-évalués. Quand la lutte pour la Palestine entra dans sa phase décisive, le Yishouv était mieux préparé, davantage mobilisé, et mieux organisé que ses adversaires régionaux. La Haganah, qui fut renommée «Forces israéliennes de défense » le 31 mai, pouvait compter sur une importante réserve d'officiers entraînés en Europe ou sur place, possédant une expérience militaire. Elle disposait d'un système de commandement et de contrôle centralisé. De surcroît, à la différence des armées des États arabes, en particulier celles de l'Irak et de l'Égypte, cette force militaire disposait de lignes de communications internes courtes, ce qui lui permettait d'agir avec un avantage en termes de vitesse et de mobilité.

Pendant la période de guerre larvée, de décembre 1947 au 14 mai 1948, le Yishouv prit progressivement l'avantage dans la lutte avec ses adversaires palestiniens. Ses forces armées étaient plus nombreuses, mieux entraînées, et plus avancées sur le plan technologique. Malgré quelques échecs initiaux, ces atouts lui permirent de gagner de façon décisive la bataille contre les Arabes palestiniens. Même lorsque les États arabes engagèrent leurs armées régulières - ce qui marqua le début de la phase de guerre déclarée -, le Yishouv garda sa supériorité numérique. À la mi-mai, les effectifs des troupes arabes, régulières et irrégulières, qui opéraient en Palestine, se situaient entre 20 et 25000 combattants. Tsahal en alignait 35000 , sans compter les troupes de deuxième ligne dans les implantations. À la mi-juillet, Tsahal mobilisa complètement 65000 hommes en armes, en septembre, jusqu'à 90000 , et en décembre le chiffre atteignit un pic de 96441 combattants. Les États arabes renforcèrent également leur contingent, mais sans pouvoir faire face à ce taux d'accroissement. Ainsi, à chaque phase de la guerre, Tsahal était en position de supériorité numérique significative sur toutes les forces arabes engagées, et pendant la phase finale de la guerre le rapport d'effectifs en sa faveur était presque de deux contre un ${ }^{29}$.

31 La grande faiblesse de Tsahal, pendant la première phase de combat en mai-juin, était relative à la puissance de feu. Les armées arabes étaient bien mieux équipées, tout spécialement en armes lourdes. Cependant, pendant la première trêve, en violation de l'embargo des Nations unies sur les armes, Israël importa du matériel venant de toute l'Europe (en particulier de Tchécoslovaquie) : fusils, mitrailleuses, blindés, fusils d'assaut, chars, avions, et toutes sortes de munitions en grande quantité. Ces acquisitions illicites d'armes permirent à Tsahal de retourner la balance en sa faveur de façon décisive. Pendant la deuxième phase du conflit, Tsahal passa à l'offensive, et pendant la troisième vague de combats elle fit face à chacune des armées arabes et les défit l'une après l'autre. Le résultat final de la guerre ne fut donc pas un miracle, mais le reflet fidèle du rapport de 
force réel entre troupes arabes et israéliennes. Dans cette guerre, comme dans la plupart des conflits, le camp le plus fort remporta finalement la victoire.

Les origines du problème des réfugiés palestiniens

Un troisième point de controverse entre historiens de la vieille école et "nouveaux " historiens concerne les origines du problème des réfugiés palestiniens. La question se pose selon les termes suivants : sont-ils partis d'eux-mêmes, ou ont-ils été mis dehors ? Régulièrement depuis 1948, les voix autorisées en Israël ont soutenu que les Palestiniens avaient quitté le pays sur les ordres de leurs propres chefs et dans l'espérance d'un retour triomphant. Les récits couchés sur le papier par les historiens de la vieille école font écho à la ligne officielle. Les représentants arabes ont affirmé avec autant d'insistance qu'Israël avait expulsé manu militari quelque 750000 Palestiniens loin de leurs maisons et qu'Israël portait par conséquent l'entière responsabilité du problème des réfugiés palestiniens. La question des origines est donc directement liée à la question de la responsabilité dans la résolution de ce problème. Les affirmations des Arabes, selon lesquelles la notion de «transfert» forcé est consubstantielle au sionisme, et selon lesquelles, en 1948, les sionistes n'ont fait que profiter des circonstances pour déplacer et déposséder les habitants arabes du pays, ont rendu cette controverse encore plus acrimonieuse.

Benny Morris, dans son livre, The Birth of the Palestinian Refugee Problem, a examiné la question avec autant d'attention, de détachement et d'objectivité qu'elle pourra probablement jamais en recevoir. Il n'a pas trouvé de preuves selon lesquelles les dirigeants arabes auraient appelé les Arabes de Palestine à quitter leurs foyers et leurs villages, ni une quelconque trace d'une campagne de radio ou de presse les incitant à fuir. Du côté israélien, il n'a trouvé aucun ordre global donné au plus haut niveau, dans le sens d'une expulsion systématique des Palestiniens. Il a donc rejeté à la fois le système d'explication des ordres arabes, et celui du vol perpétré par l'État juif. Sa conclusion que l'on cite souvent est que «le problème des réfugiés palestiniens est né de la guerre, non d'un dessein, juif ou arabe. Il fut essentiellement une conséquence dérivée des peurs et des longs et difficiles combats qui caractérisèrent la première guerre israélo-arabe ; dans une moindre mesure, il fut la création délibérée des commandants militaires et des hommes politiques arabes et juifs ${ }^{30} »$. Benny Morris a déjà répondu en détail aux critiques de Teveth, et il ne servirait à rien de rendre compte ici et au coup par coup de la querelle ${ }^{31}$. Il me semble toutefois que la position de Teveth sur les origines du problème des réfugiés palestiniens est à peu près aussi subtile que le vieux dicton, haya ness vehem nassu - il y eut un miracle et ils s'enfuirent. Quiconque est prêt à admettre cela pourra avaler n'importe quoi.

Une autre catégorie de détracteurs du livre de Benny Morris est constituée d'orientalistes israéliens. Certains d'entre eux, tel Yehoshua Porath, ont été enthousiastes. D'autres, comme Asher Susser, Emmanuel Sivan et Avraham Sela, ont écrit dans une veine plus critique, tout en accordant à l'ouvrage le crédit qui lui est dû. Le reproche récurrent de ces milieux académiques est que Morris a fait très peu usage de sources arabes. En réponse à ces critiques, Morris a posé une question: la consultation du matériau documentaire arabe mentionné par ses critiques aurait-elle produit une révision fondamentale de l'analyse de l'exode palestinien, ou ajouté significativement à la description de cet exode, tel qu'il est présenté dans son livre ${ }^{32}$ ? Avraham Sela admet que l'usage des sources arabes n'aurait probablement pas changé les conclusions principales de l'étude de Morris sur les causes de l'exode palestinien. Il poursuit toutefois en arguant 
que la négligence des sources arabes disponibles et le fait de s'appuyer de façon aussi marquée sur les sources israéliennes peuvent produire un tableau déséquilibré ${ }^{33}$.

Alors qu'un autre groupe d'orientalistes soutient que Morris a attaché trop d'importance aux actions israéliennes, par rapport à d'autres facteurs, dans son explication des origines du problème des réfugiés palestiniens, de nombreux autres critiques ont estimé que les conclusions de Morris permettaient encore à Israël de s'en tirer assez bien. Une observation, fréquemment faite dans les commentaires d'auteurs occidentaux ou palestiniens, est que les preuves présentées dans le livre suggèrent un degré de responsabilité israélienne bien supérieur à ce qui est admis en conclusion ${ }^{34}$. Néanmoins, en dépit des faiblesses de sa conclusion, l'ouvrage de Morris reste une contribution remarquable, originale, scientifique, et très importante pour l'étude d'un problème clé du conflit israélo-arabe.

Les relations israélo-jordaniennes

Un quatrième enjeu, source de vive controverse en Israël, est la nature des relations israélo-jordaniennes et, plus spécifiquement, l'affirmation d'une collusion, ou du moins d'un accord tacite, entre le roi Abdallah et l'Agence juive entre 1947 et 1949. L'existence d'une connivence entre ces deux parties est depuis un certain temps de notoriété publique et les deux rencontres entre Golda Meir et Abdallah en novembre 1947 et mai 1948 ont même été mises en scène dans des films populaires. L'accusation de collusion n'est pas nouvelle non plus. Elle a été lancée dans un livre publié par le colonel Abdallah al-Tall, qui avait servi de messager entre Abdallah et la partie juive, après son coup d'État manqué et sa défection au service de l'Égypte ${ }^{35}$. Une mise en cause du même ordre a pesé contre Ben Gourion dans le livre que le lieutenant-colonel Israël Baer a rédigé en prison après sa condamnation pour espionnage au service de l'Union soviétique ${ }^{36}$. Abdallah alTall condamnait la trahison d'Abdallah envers ses frères arabes et son abandon intéressé des Palestiniens. Baer jugeait Ben Gourion coupable d'un pacte diabolique avec les forces de la réaction arabe et de l'impérialisme britannique. Une kyrielle de livres et d'articles sur les relations entre sionistes et hachémites ont en outre été composés par des universitaires israéliens, dont les plus récents sont ceux de Dan Schueftan et Uri BarJoseph $^{37}$. Pourtant, dans la dernière moisson d'ouvrages sur ces relations bilatérales assez peu ordinaires, c'est mon ouvrage Collusion across the Jordan qui est passé à la notoriété des deux côtés du Jourdain et a été pris pour cible par les historiens de la « vieille école ». La thèse centrale que je soutiens dans ce livre est qu'un accord oral a été passé en novembre 1947 entre Abdallah et l'Agence juive dans le sens d'une partition de la Palestine entre la Transjordanie et l'État juif à naître, au terme du mandat britannique ; et que cet accord a constitué la base d'une politique de retenue mutuelle pendant la guerre israélo-arabe et du maintien de la collaboration après-guerre. Une dernière thèse du livre affirme que la Grande-Bretagne connaissait et approuvait cet accord secret entre Hachémites et sionistes pour une partition qui ne suivrait pas les lignes du plan de partage de l'ONU.

38 Cette idée remet en cause la vision traditionnelle du conflit israélo-arabe comme problème tout au plus bipolaire, dans lequel un monde arabe monolithique et animé d'une hostilité implacable s'oppose aux Juifs. Elle suggère au contraire que les dirigeants arabes étaient profondément divisés sur la façon de régler la question sioniste, et que l'un d'entre eux était favorable à un accord avec l'Agence juive pour un partage de la Palestine aux dépens des Palestiniens. Cette thèse sape également la version héroïque qui représente Israël cerné par une chaîne ininterrompue d'ennemis arabes et contraint de 
repousser une attaque concertée sur tous les fronts. Sans surprise, l'histoire officielle de la guerre d'Indépendance néglige ne serait-ce que de faire mention de l'accord informel avec Abdallah ${ }^{38}$. Lors même que cette entente est admise, la ligne officielle est qu'Abdallah est revenu sur ses engagements au moment critique, et que cet épisode n'eut pas d'influence, ou seulement de façon marginale, sur le déroulement de la guerre ${ }^{39}$.

Ressassant la ligne officielle, Shabtai Teveth nie avec véhémence que les dirigeants juifs aient jamais été impliqués dans une telle collusion, ou aient eu un allié du côté arabe. Il admet en grimaçant qu' « Israël et la Jordanie maintinrent un certain dialogue », mais soutient ensuite que " tout au plus, il n'y avait entre eux qu'un accord de convenance... Il n'y eut rien, dans une telle entente, qui pût suggérer une collusion destinée à tromper une tierce partie, en l'occurrence les Arabes palestiniens ${ }^{40}$ ». Là encore, quiconque croit cela est d'une crédulité sans limites. Si tout ce qui passait entre Israël et la Jordanie était un dialogue, il devait s'agir d'un dialogue d'un genre bien curieux, puisqu'il dura trente ans, qu'il fut tenu dans la clandestinité, qu'il concernait un rival commun, et qu'en conséquence de l'argent passait d'une poche à l'autre. Le dialogue fut interrompu entre mai et août 1948, cela est hors de doute. Pourtant, si l'on considère cette relation sur le long terme, certainement les termes de "partenariat stratégique", voire de "pacte diabolique » seront plus adéquats que celui de dialogue.

Teveth est, de toute évidence, si infatué de la doctrine de l'immaculée conception d'Israël qu'il est absolument imperméable à toute preuve allant en sens contraire. Il a tranché la question une bonne fois pour toutes, et il ne veut pas être troublé par les faits. Son article fournit un bon exemple de l'absurde prolixité dont les historiens de la vieille école sont capables afin de réprimer des vérités déplaisantes sur la venue au monde d'Israël. À en juger par les normes brutales des relations internationales, le jeu de l'intérêt et du hasard entre les sionistes et le souverain hachémite n'était ni extraordinaire ni spécialement répréhensible. Les deux parties agirent avec pragmatisme dans leur propre intérêt. Le problème ne survient que lorsque l'on considère l'affirmation selon laquelle l'attitude d'Israël se fondait sur la morale plutôt que sur l'intérêt bien compris.

Les relations entre la Transjordanie et Israël dans la guerre de 1948 ont été récemment passées en revue par Avraham Sela dans un article de soixante-six pages dans les Middle Eastern Studies. L'utilisation d'archives par Sela et son examen exhaustif de la littérature sur le sujet, en arabe tout particulièrement, en font une contribution précieuse à l'historiographie de la guerre de 1948. Cela étant, aucun des arguments que j'ai présentés dans Collusion across the Jordan ne me paraît invalidé. La thèse de Sela est que « les conditions et les hypothèses de base qui avaient constitué les fondements de l'accord oral entre Abdallah et l'Agence juive au sujet du partage de la Palestine, selon des termes fixés dès l'été 1946, ont été modifiées si profondément pendant la phase de guerre nondéclarée (décembre 1947-mai 1948) que le contenu de l'entente était dépassé et inapplicable ${ }^{41} »$.

Je pense que, malgré toutes les transformations, le premier accord et la longue histoire de la coopération - qui remonte à la fondation de l'émirat de Transjordanie en 1921 - ont continué à influer sur l'attitude des deux camps pendant la guerre. Sela affirme que, pendant la première phase de la guerre, les deux parties, et en particulier les Israéliens, se sont comportées selon l'adage "à la guerre comme à la guerre ". Même si cette conclusion est valide en ce qui concerne Israël, je soulignerais qu'elle est sans validité pour la Jordanie. Bien que l'accord n'ait plus été contraignant et que les contacts aient été rompus, chaque camp - et spécialement la Transjordanie - a continué à poursuivre des 
buts limités, et à agir avec modération à l'égard de l'autre jusqu'à la fin de la guerre. Quoiqu'ils soient devenus ennemis au plus fort du conflit, ils sont demeurés, pour citer la phrase très juste d'Uri Bar-Joseph, « les meilleurs ennemis ».

En somme, Sela nous dit que la guerre est un phénomène complexe et difficile à démêler. Sans contredit. Une des raisons en est que la guerre fait entrer en ligne de compte à la fois la politique et l'usage de la force. L'historiographie de la vieille école se préoccupe essentiellement des aspects militaires de la guerre. Je me suis efforcé de rétablir un certain équilibre en considérant le côté politique, et tout particulièrement les interactions entre politique et stratégie. Sela avance encore que « le mythe de la collusion suppose que l'on fasse l'hypothèse selon laquelle l'acceptation commune par les sionistes et les Palestiniens du plan de partage et sa réalisation pacifique étaient possibles ${ }^{42} \%$. Je ne postule rien de tel. Au contraire, précisément parce que les Palestiniens ont rejeté la partition, je considère la collaboration entre Abdallah et l'Agence juive comme une stratégie raisonnable et réaliste pour chacune des parties concernées. En d'autres termes, je considère que, pendant la période 1947-1949, Israël n'avait pas d'option palestinienne, ni aucune autre option arabe, à l'exception de la piste jordanienne. Le roi Abdallah était le seul chef d'État arabe prêt à accepter le principe d'un partage et la coexistence pacifique avec un État juif, après que les poussières de la guerre seraient retombées. De mars à avril 1948, cet accord fut mis à rude épreuve par la poursuite de l'offensive juive. Dans la période allant de mai à juillet 1948, les deux parties en vinrent aux mains. Du point de vue d'Abdallah, dans la perspective de la conciliation de l'après-guerre, ce n'était qu'une fitna, une querelle de famille, et les Juifs avaient commencé. Après l'explosion de violence initiale, les deux camps commencèrent à dégager leurs poings, ainsi que cela se produit dans ce type de disputes.

Reste à savoir si le terme "collusion» est approprié pour décrire les relations entre Abdallah et l'Agence juive, puis l'État d'Israël. Certaines des critiques du livre ont porté sur son titre plutôt que sur son contenu. C'est pour cette raison que dans la version abrégée et révisée parue en livre de poche, j'ai adopté un titre plus neutre, The Politics of Partition $^{43}$. Dans la préface de cette nouvelle édition, j'explique que, tout en ayant abandonné le terme dérangeant du titre, j'étais toujours d'avis que le lien entre Israël et la Jordanie relevait, au moins sur certains points, de la «collusion »: «Cela se passait sous un fin voile de secret; son existence était niée avec ferveur par ceux qui y participaient ; c'était dirigé contre une tierce partie ; cela impliquait une bonne dose de manigances en sous-main et de cabales; et c'était consciemment et délibérément destiné à mettre sous le boisseau la volonté de la communauté internationale, exprimée par l'Assemblée générale des Nations unies, en faveur de la création d'un État arabe indépendant sur une partie de la Palestine ${ }^{44}$.» À la réflexion, je regrette plutôt d'avoir changé le titre de mon livre. Le titre original était bien vu. La collusion est un terme qui en vaut bien d'autres pour décrire les relations souterraines entre le souverain hachémite et le mouvement sioniste pendant la période 1921-1951, en dépit du violent intermède de l'été 1948.

Les buts de guerre arabes

En lien étroit avec les relations israélo-jordaniennes se pose le problème des buts de guerre arabe en 1948, cinquième pomme de discorde entre la vieille école historique et les nouveaux historiens. La question est la suivante : pourquoi les États arabes ont-ils envahi la Palestine avec leurs armées régulières le jour de l'expiration du mandat britannique et de la proclamation de l'État d'Israël? La réponse sioniste traditionnelle est que la 
motivation de l'invasion était de détruire l'État juif naissant et de jeter les Juifs à la mer. La réalité est plus complexe.

Il est vrai que tous les États arabes, à l'exception de la Jordanie, ont rejeté le plan de partition de l'ONU. Il est exact que sept armées arabes envahirent la Palestine dès le lendemain de la proclamation de l'État d'Israël. Il est vrai que l'invasion fut accompagnée de rhétorique sanguinaire et de menaces de jeter les Juifs à la mer. Il est juste que, en plus des armées arabes régulières et de l'Armée de guerre sainte du grand mufti de Jérusalem, divers groupes de volontaires arrivèrent en Palestine, le plus important étant l'Armée de libération arabe, soutenue par la Ligue arabe et dirigée par l'aventurier syrien Fawzi alQawuqji. Il est vrai, et plus important, que les experts militaires de la Ligue arabe avaient élaboré un plan unifié d'invasion et que ce plan était d'autant plus dangereux qu'il avait des objectifs plus limités et plus réalistes que ceux que supposait la rhétorique échevelée du panarabisme.

Cependant, le roi Abdallah, qui reçut le commandement nominal de toutes les forces arabes en Palestine, mit en pièces ce plan par des changements de dernière minute. Ses objectifs, en envoyant son armée en Palestine, n'était pas d'empêcher la création d'un État juif, mais de se rendre maître de la partie arabe de la Palestine; ce qui supposait d'empêcher la fondation d'un État palestinien indépendant. Dans la mesure où les Palestiniens n'avaient à peu près rien fait pour créer un tel État, la partie arabe de la Palestine serait quand même probablement passée sous le contrôle d'Abdallah sans manigances ni cabales, mais c'est encore un autre problème. Ce qui est clair, c'est que, sous le commandement de Glubb Pacha ${ }^{45}$, la Légion arabe fit tous les efforts possibles pour éviter une collision frontale et, à l'exception d'un ou deux incidents, ne fit aucune tentative pour empiéter sur le territoire alloué par les cartographes de l'ONU à l'État juif.

L'amour n'entourait pas les relations entre Abdallah et les autres chefs d'État arabes, qui le soupçonnaient d'être de mèche avec l'ennemi. Abdallah fut toujours une sorte de paria aux yeux du reste du monde arabe, et son amitié pour les Juifs n'y était pas pour peu. La Syrie et le Liban se sentaient menacés par sa vieille ambition de se rendre maitre de la Grande Syrie. L'Égypte, qui menait le bloc anti-hachémite à l'intérieur de la Ligue arabe, se sentait également menacée devant les plans d'expansion en Palestine que nourrissait Abdallah. Le roi Farouk prit la décision d'intervenir en Palestine au dernier moment, contre l'avis de ses experts civils et militaires, et ce, à tout le moins, pour contrer la montée en puissance de son rival. Par conséquent, les raisons de l'invasion de la Palestine étaient plutôt mal assorties. Il n'y eut d'ailleurs pas de plan d'action arabe unique pendant la guerre de 1948. Au contraire, c'est l'incapacité des Arabes à coordonner leurs programmes diplomatiques et militaires qui fut largement responsable du désastre qui les emporta. L'objectif affirmé de l'invasion arabe, à savoir venir au secours des Palestiniens pris dans la guerre, fut précisément celui qui ne fut pas atteint. Nulle part, la distance entre la rhétorique panarabe et la réalité ne fut plus grande qu'au sujet des Arabes palestiniens ${ }^{46}$. La réalité était celle de l'égoïsme national, et chaque État arabe poursuivait ses propres buts. Ce qui était censé être une guerre sainte contre les Juifs devint rapidement une rivalité générale pour occuper le sol. La division et la discorde couraient dans les rangs de l'improbable coalition arabe, et devinrent plus profondes à chaque nouvelle défaite. Les dirigeants israéliens connaissaient ces divisions et les exploitèrent au maximum. Ainsi, ils lancèrent une offensive contre l'armée égyptienne en octobre, et à nouveau en décembre 1948, avec le ferme espoir que leur vieil ami d'Amman ne s'en mêlerait pas. Les historiens de la «vieille école», en se concentrant presque 
exclusivement sur les opérations militaires de 1948, aboutirent à l'image familière d'une guerre israélo-arabe dans laquelle les Arabes se retrouvaient tous derrière un but unique, et où tous étaient désireux de défaire et de détruire Israël. Rétrospectivement, pourtant, la configuration politique du camp arabe en 1948 apparaît bien plus compliquée, et les motivations de l'invasion de la Palestine tout autrement variées.

La paix fuyante

Le dernier, mais non le moindre, des contentieux dans le débat entre la vieille école et les nouveaux historiens est la question de savoir pourquoi la paix s'est révélée impossible à atteindre après la première guerre israélo-arabe. Au cœur de l'interprétation de la vieille école se trouve la notion d'intransigeance arabe. Selon cette version, Israël a déployé des trésors d'activité pour obtenir une solution de paix au conflit, mais tous ses efforts se sont heurtés à l'écueil de l'intransigeance arabe. Les nouveaux historiens pensent que l'Israël de l'après-guerre étaient plus intransigeant que les États arabes et qu'en conséquence cet État porte une grande part de responsabilité dans le cul-de-sac où l'on s'est retrouvé après la cessation officielle des hostilités ${ }^{47}$.

Les preuves qui appuient la nouvelle interprétation proviennent pour l'essentiel des dossiers du ministère israélien des Affaires étrangères. Ceux-ci sont un filon de preuves des signes de paix des Arabes et de leur disposition à négocier avec Israël dès septembre 1948. Les deux thèmes centraux de la dispute étaient les réfugiés et les frontières. Chacun des États arabes s'était préparé à négocier directement avec Israël et à marchander sur l'un et l'autre enjeu.

51 Abdallah proposa un projet de solution politique globale avec Israël en échange de certaines concessions territoriales, en particulier un couloir reliant la Jordanie à la Méditerranée, qui lui aurait permis de rembarrer ses critiques arabes qui l'accusaient de faire une paix séparée avec Israël. Le colonel Husni Zaim, qui prit le pouvoir en Syrie en mars 1949 et fut renversé quatre mois plus tard, offrit à Israël une paix totale avec échange d'ambassades, relations économiques normales et l'installation de 300000 réfugiés palestiniens en Syrie contre un ajustement faisant passer la frontière entre les deux pays au milieu du lac de Tibériade ${ }^{48}$. Le roi Farouk d'Égypte demanda la cession de Gaza

52 et d'une substantielle bande de désert le long du Sinaï pour prix de la reconnaissance de facto d'Israël. Les trois chefs d'État arabes firent tous preuve d'un remarquable pragmatisme dans leur approche des relations avec l'État juif. Ils étaient même impatients de se devancer mutuellement, car ils supposaient que celui qui, le premier, aboutirait à une solution avec Israël, en obtiendrait les meilleurs termes. Zaim déclara ouvertement son intention d'être le premier dirigeant arabe à faire la paix avec Israël.

Dans chacun de ces cas, et quoique pour des raisons légèrement différentes à chaque fois, David Ben Gourion considéra que le prix demandé pour la paix était trop élevé. Il était prêt à faire la paix sur la base du statu quo ; il se refusait à s'engager sur le chemin d'une paix qui impliquait plus que des concessions minuscules, de la part d'Israël, que ce soit sur les réfugiés ou sur le tracé des frontières. Ainsi que le révèlent ses Carnets, il considérait que les accords d'armistice avec les États arabes voisins satisfaisait Israël en matière de reconnaissance, de sécurité et de stabilitée ${ }^{49}$. Il savait qu'Israël devrait payer des traités de paix formelle par la cession de morceaux substantiels de territoires et par le retour d'un nombre important de réfugiés palestiniens, et il ne considérait pas que le jeu en valait la chandelle. Savoir si Ben Gourion a fait le bon choix est une question d'opinion personnelle. Le fait indéniable est qu'il avait le choix. 
54 La controverse entourant la paix manquée est examinée dans un livre d'Itamar Rabinovich, ancien recteur de l'université de Tel-Aviv et l'un des meilleurs experts israéliens sur la politique arabe contemporaine. Le titre du livre est inspiré du poème de Robert Frost : "The Road Not Taken : Early Arab-Israeli Negotiations ». Ce titre implique que l'échec de ces pourparlers n'était pas inévitable, qu'il y avait un autre chemin vers la paix - celui qui n'a pas été emprunté. Cependant, le livre ne propose aucune thèse et ne s'engage pas directement dans le débat entre la vieille école et les nouveaux historiens. Rabinovich préfère rester au-dessus de la mêlée. Il est si réticent à l'idée de distribuer les reproches que son livre se finit sans conclusion explicite. Tout ce qu'il se permet de dire est que « les choix de 1948-1949 furent faits par les Arabes, les Israéliens, les Américains et les autres. Tout le mérite ou toute la responsabilité de ces décisions appartiennent à l'ensemble de ces parties ${ }^{50}$ ». La conclusion implicite de Rabinovich est néanmoins que, en raison de l'instabilité des régimes arabes, le refus de Ben Gourion d'assumer le moindre risque dans l'intérêt de la paix était justifié. Pourtant, sur chacune des questions cruciales, la présentation de Rabinovich sape les fondements de l'affirmation des historiens de la "vieille école", selon laquelle Israël dut faire face à une totale intransigeance arabe, et confirme l'argumentaire révisionniste qui soutient que l'intransigeance israélienne fut l'obstacle le plus sérieux posé à la conclusion de la paix ${ }^{51}$. Conclusion

55 Cet article examine la version sioniste de la vieille école de la première guerre israéloarabe et sa remise en cause par la nouvelle historiographie. Ma conclusion est que la version traditionnelle comporte des failles profondes, et qu'une révision radicale est nécessaire, à la lumière des nouvelles informations disponibles. Pour le dire brutalement, cette première version ne s'élève pas beaucoup plus haut que la propagande des vainqueurs. Le débat entre la vieille école historiographique et la nouvelle, au surplus, n'est pas seulement d'intérêt historique. Il va droit au cœur de l'image de soi que se donne Israël. C'est la raison pour laquelle la querelle des historiens a suscité un profond intérêt du public et de si fortes passions politiques.

56 Le débat autour de 1948 ressemble au débat américain sur les origines de la guerre froide. Ce dernier a évolué par stades. Pendant les années 1950, le point de vue prétendument traditionaliste tint bon. Selon cette conception, l'expansionnisme soviétique était responsable de la guerre froide, cependant que la politique américaine était essentiellement une réponse et une défense. Par la suite, dans le contexte de la guerre du Viêt-nam et de la crise de confiance américaine qui l'accompagnait, une école révisionniste émergea, composée essentiellement de chercheurs plus jeunes et orientés à gauche. Selon eux, la guerre froide était le résultat des progrès du capitalisme américain, et c'est l'Union soviétique qui eut une attitude défensive. À la suite de l'ouverture des archives, une troisième école de pensée vit le jour, l'école post-révisionniste. Le réexamen des hypothèses et des arguments des deux premières écoles à la lumière d'une nouvelle documentation engendra une nouvelle synthèse. La marque de fabrique du postrévisionnisme est son attitude qui ne fait porter le blâme ni sur l'un ni sur l'autre camp, mais s'efforce de comprendre la dynamique du conflit que nous appelons la guerre froide.

Le débat sur les origines du conflit israélo-arabe semble suivre un modèle similaire. Une école traditionaliste, composée d'acteurs du conflit et de partisans aussi bien que d'historiens proches des franges installées du monde politique, a mis la totalité de la responsabilité de la guerre de 1948 et de ses conséquences sur le compte des Arabes. Après quoi, et à la suite de l'ouverture des archives, une nouvelle école, principalement 
composée d'historiens marqués à gauche, entreprit de réinterpréter les événements qui ont conduit à la création de l'État d'Israël. Ces derniers adoptent des positions nettement plus critiques sur la conduite d'Israël dans les années 1947-1949, et lui attribue une part de responsabilité plus grande dans la naissance du problème des réfugiés palestiniens et dans la formation de l'impasse politique qui dure jusqu'à ce jour au Proche-Orient. Le débat entre vieille école et nouveaux historiens est amer et plein d'acrimonie, et il se déroule dans une atmosphère très fortement politisée. Il est dommage de devoir ajouter qu'aucune synthèse post-révisionniste n'est en vue. Les batailles entre historiens, comme les vraies batailles, ne se laissent pas détourner de leur cours.

\section{NOTES}

1. Émile Habibi, Al-Waqẩi al-Gharrîba fí Ikhtifầ'Sa'îd Abî al-Nahs al-Mutashẩil (Les Aventures de Sa îd le Peptimiste), Beirut, Dâr Ibn Khaldûn, 1974, p. 37. N.d.T. : Le néologisme " peptimiste ", qui nous a semblé le mieux convenir, est la contraction de pessimiste et d'optimiste.

2. Ibid., p. 35.

3. Simha Flapan, The Birth of Israel : Myths and Realities, New York, Pantheon, 1987, p. 8.

4. Ibid., p. 10.

5. Cambridge, Cambridge University Press, 1988.

6. Paris, La Fabrique, 2000.

7. Avi Shlaim, Oxford, Clarendon Press, 1988.

8. Désignées en français, et dans la suite de l'article, par leur acronyme hébreu : Tsahal, (N.d.T.).

9. Israel Baer, Bitahon Israel : Etmol, Hayom, Mahar (La Sécurité d'Israël : Hier, aujourd'hui, demain), Tel-Aviv, Amikam, 1966.

10. Gavriel Cohen, « Hamediniyut Habritit Erev Milhemet Ha'atzam'ut », dans Yehuda Wallach (éd.), Hayinu Keholmim, Givatayim, Massada, 1985.

11. Yaacov Shimoni, « Ha'aravim Likrat Milhemet Israel-'Arav, 1945-1948 » (Les Arabes et la montée vers la guerre avec Israël, 1945-1948), Hamizrah Hehadash 47, 3, 1962.

12. Meir Pa'il, «Hafqa'at Haribonut Hamedinit shel Filastin miyedei Hafalestinim »

(L'éviction des Palestiniens au droit à la souveraineté politique sur la Palestine), Ziyonut 3, 1973.

13. A Political Study of the Arab-Jewish Conflict: The Arab Refugee Problem, Genève, Librairie E. Droz, 1959.

14. A. Yisra'eli (pseudonyme de Moshe Machover et Akiva Orr), Shalom, Shalom - ve'ein Shalom : Yisra'el-Arav, 1948-1961 (La Paix, la paix - et il n'y a pas de paix : Israël et les Arabes, 1948-1961), Jérusalem, s. n., 1961.

15. Archives de l'État d'Israël et Archives centrales sionistes, Gedalia Yogev (éd.), Political and Diplomatic Documents, December 1947-May 1948, Jérusalem, Presses du gouvernement d'Israël, 1980 ; Archives de l'État d'Israël, Yehushua Freundlich (éd.), Documents on the Foreign Policy of Israel, May-September 1948, vol. 1, Jérusalem, Presses du gouvernement d'Israël, 1984 : Yemima Rosenthal (éd.) Documents on the Foreign Policy of Israel : Armistice 
Negotiations with the Arab States, December 1948-July 1949, vol. 3, Jérusalem, Presses du gouvernement d'Israël, 1986.

16. Gouvernement d'Irak, Taqrîr Lajnat al-Tahqî̀ al-Niyâbiyya fî Qadiyyat Filastîn (Rapport sur les commissions parlementaires d'enquêtes sur la défaite de Palestine), Bagdad, s. n., 1949.

17. Références dans Walid Khalidi, «The Arab Perspective », dans William Roger Louis \& Robert W. Stookey (éd.), The End of the Palestine Mandate, Londres, I. B. Tauris, 1986.

18. Pour une recension de cette littérature, voir Avraham Sela, « Arab Historiography of the 1948 War : The Quest for Legitimacy ", dans Lawrence J. Silberstein (éd.), New Perspectives on Israeli History: The Early years of the State, New York : New York University Press, 1991.

19. Benny Morris, «The New Historiography : Israel Confronts Its Past », dans Tikkun, 3, 6, novembre-décembre 1988. Cet article très discuté est réimprimé dans Benny Morris, 1948 and After: Israel and the Palestinians, Oxford, Clarendon Press, 1990.

20. Une historienne du sionisme, Anita Shapira, fut incitée par l'affirmation de Menachem Begin à entamer le réexamen de l'éthos de défense du sionisme, à travers la période pré-étatique. Voir Tom Segev, "The Anguish of Poor Samson », dans Ha'aretz, 16 octobre 1992 ; Anita Shapira, Land and Power : The Zionist Resort to Force, 1881-1948, New York, Oxford University Press, 1992, p. VII.

21. Shabtai Teveth, «Charging Israel with Original Sin », dans Commentary, septembre 1989 , p. 33.

22. Ibid., p. 25.

23. Ibid.

24. Benny Morris, dans Ha'aretz, 9 mai 1989 ; idem, « The Eel and History : A Reply to Shabtai Teveth ", dans Tikkun, 5, 1, janvier-février 1990 ; idem, dans 1948 and After, pp. 27-29.

25. Voir ma lettre au rédacteur en chef, dans Commentary, février et juillet 1990.

26. Le Yishouv désigne la population juive de Palestine, (N.d.T.).

27. Ilan Pappé, La Guerre de 1948 en Palestine; Shlaim, Collusion across the Jordan; idem, «Britain and the Arab-Israeli War of 1948 », dans Journal of Palestine Studies, 16, 4, été 1987. Sur la théorie selon laquelle les Britanniques voulaient réduire la part juive de la Palestine à un «moignon d'État ", voir William Roger Louis, The British Empire in the Middle East, 1945-1951: Arab Nationalism, the United States, and Postwar Imperialism, Oxford, Clarendon Press, 1984, pp. 372-379.

28. Hajj Amin al-Husayni, grand mufti de Jérusalem, a incarné la tendance intransigeante du nationalisme palestinien à la tête du Haut Comité arabe. Ėxilé en 1939 par les Britanniques à la suite de la révolte arabe de 1936-1939, le mufti était ennemi de Londres et s'était allié aux pays de l'Axe pendant la Seconde Guerre mondiale. Il était en exil au Liban pendant la guerre de 1948, et tenta de s'opposer au mouvement sioniste et à la politique d'Abdallah de Jordanie pendant la guerre en lançant la guerre sainte contre le sionisme et les Juifs de Palestine, et en levant une armée parmi ses partisans arabes palestiniens (N.d.T.).

29. Simha Flapan, op. cit., "Sixième mythe ", en particulier le tableau de trois estimations différentes des effectifs de troupes, p. 196 ; Benny Morris, 1948 and After, pp. 13-16. Une étude fondée sur un accès privilégié aux documents de Tsahal confirme la ligne révisionniste en montrant que l'embargo sur les armes des Nations unies frappait beaucoup plus durement les Arabes que Tsahal : Amitzur Ilan, The Origins of the Arab-Isareli Arms Race, à paraître. 
30. Benny Morris, The Birth of the Palestinian Refugee Problem, 1947-1949, p. 286.

31. En plus des articles dans Ha'aretz et Commentary, Teveth a publié « The Palestine Arab Refugee Problem and Its Origins », dans Middle Eastern Studies, 26, 2, avril 1990.

32. Benny Morris dans Ha'aretz des 23 avril et 1er mai 1992.

33. Avraham Sela dans Ha'aretz des 4 et 11 octobre 1991.

34. Voir, par exemple, Michael Palumbo, « What Happened to Palestine ? The Revisionists Revisited ", dans The Link, 23, 4, septembre-octobre 1990 ; Rashid Khalidi, « Revisionist Views of the Modern History of Palestine : 1948 ", dans Arab Studies Quarterly, 10, 4, automne 1988 ; Ibrahim Abu-Lughod, «The War of 1948 : Disputed Perspectives and Outcomes ", dans Journal of Palestine Studies 18, 2, hiver 1989 ; Nur Masalha, Expulsion of the Palestinians : The Concept of «Transfer » in Zionist Political Thought, 1882-1948, Washington D.C., Institute for Palestine Studies, 1992.

35. Abdallah al-Tall, KârithatFilastîn : Mudhakkirât 'Abdullâh at-Tall, Qâ'id Ma'rakat alQuds (La catastrophe palestinienne : les mémoires d'Abdallah al-Tall, général de la bataille de Jérusalem), Le Caire, Dar al-Qalam, 1959.

36. Op. cit.

37. Dan Schueftan, Optzya Yardenit : Israel, Yarden Vehapalestinim (L'option jordanienne : Israël, la Jordanie et les Palestinens), Hakkibutz Hame'uhad, Yad Tabenkin, 1986 ; Uri BarJoseph, The Best of Enemies : Israel and Transjordan in the War of 1948, Londres, Frank Cass, 1987.

38. Tsahal, Toldot Milhemet Hakomemiyut (Histoire de la guerre d'Indépendance), Tel-Aviv, Ma'aracbot, 1959.

39. Par exemple, dans l'entretien de cet auteur avec Yigael Yadin, chef d'état-major en titre en 1948, dans Avi Shlaim, op. cit., p 236.

40. Shabtai Teveth, « Charging Israel with Original Sin », p. 28.

41. Avraham Sela, « Transjordan, Israel and the 1948 War : Myth, Historiography and Reality ", dans Middle Eastern Studies, 28, 4, octobre 1992, p. 627.

42. Ibid., p. 680.

43. Avi Shlaim, The Politics of Partition : King Abdullah, the Zionists and Palestine, 1921-1951, Oxford, Oxford University Press, 1990.

44. Ibid., p. VIII.

45. Sir John Glubb, officier britannique, commandait la Légion arabe, que lui et d'autres officiers-conseillers envoyés par le Royaume-Uni avaient contribué à former et à moderniser.

46. Voir, par exemple, Avi Shlaim, «The Rise and Fall of the All-Palestine Government in Gaza », dans Journal of Palestine Studies, 20, 1, automne 1990.

47. Simha Flapan, op. cit., "Septième mythe "; Avi Shlaim, Collusion across the Jordan; Benny Morris, 1948 and After, pp. 22-27 ; et Ilan Pappé, op. cit., chapitres 8-10.

48. Avi Shlaim, « Husni Zaim and the Plan to Resettle Palestinian Refugees in Syria », dans Journal of Palestine Studies, 15, 4, été 1986.

49. David Ben Gourion (Gershon Rivlin \& Elhanan Orren [éd.]), Yoman Hamilhama (Carnets de guerre), vol. 3, Tel-Aviv : ministère de la Défense, 1982, p. 993.

50. Itamar Rabinovich, The Road Not Taken : Early Arab-Israeli Negotiations, New York, Oxford University Press, 1991, p. VIII.

51. Pour une critique en détail de Rabinovich, voir Benny Morris, «A Second Look at the « Missed Opportunity », or Smoothing Out History : A Review Essay », dans Journal of Palestine Studies, 24, 1, automne 1994. 


\section{AUTEUR}

AVI SHLAIM

Avi Shlaim occupe la chaire Alastair Buchan de relations internationales à l'université d'Oxford, et est fellow au St Antony's College, Oxford. 